\title{
Fluvoxamine for fatigue in primary biliary cirrhosis and primary sclerosing cholangitis: a randomised controlled trial [ISRCTN88246634]
}

\author{
Pieter CJ ter Borg*1, Erik van Os², Walter W van den Broek², \\ Bettina E Hansen ${ }^{3}$ and Henk R van Buuren ${ }^{1}$
}

\begin{abstract}
Address: ${ }^{1}$ Department of Gastroenterology and Hepatology, Erasmus MC, Dr. Molewaterplein 40, Rotterdam, The Netherlands, ${ }^{2}$ Department of Psychiatry, Erasmus MC, Dr. Molewaterplein 40, Rotterdam, The Netherlands and ${ }^{3}$ Department of Epidemiology and Biostatistics, Erasmus MC, Dr. Molewaterplein 40, Rotterdam, The Netherlands

Email: Pieter CJ ter Borg* - pterborg@zonnet.nl; Erik van Os - e.vanos@erasmusmc.nl; Walter W van den

Broek -w.vandenbroek@erasmusmc.nl; Bettina E Hansen - b.hansen@erasmusmc.nl; Henk R van Buuren - h.vanbuuren@erasmusmc.nl

* Corresponding author
\end{abstract}

Published: 13 July 2004

BMC Gastroenterology 2004, 4:13 doi:I0.1 186/147I-230X-4-13

Received: 14 April 2004

Accepted: 13 July 2004

This article is available from: http://www.biomedcentral.com//47/-230X/4/13

(C) 2004 ter Borg et al; licensee BioMed Central Ltd. This is an Open Access article: verbatim copying and redistribution of this article are permitted in all media for any purpose, provided this notice is preserved along with the article's original URL.

\begin{abstract}
Background: Fatigue is a major clinical problem in many patients with primary biliary cirrhosis (PBC) and primary sclerosing cholangitis (PSC). An effective treatment has not been defined. Recently, a large proportion of patients with these diseases was found to have symptoms of depression. Because fatigue is a frequent symptom of depression and there is some evidence that treatment with an antidepressant improves fatigue in patients with fibromyalgia, we hypothesised that the antidepressant fluvoxamine might improve fatigue related to PBC and PSC.
\end{abstract}

Methods: Fatigued patients were randomised to receive fluvoxamine (75 mg BID) or placebo for a six-week period. Fatigue and quality of life were quantified using a visual analogue scale, the Fisk Fatigue Severity Scale, the Multidimensional Fatigue Inventory and the SF-36.

Results: Seventeen and 16 patients were allocated to fluvoxamine and placebo, respectively. There was no statistically significant beneficial effect of fluvoxamine on fatigue or quality of life. The median VAS scores in the fluvoxamine and placebo groups were 7.40 and 7.45 at day $0,6.9$ and 7.15 at day $14,7.45$ and 7.65 at day 42 and 7.8 and 8.0 four weeks after treatment discontinuation.

Conclusion: We found no evidence for a beneficial effect of fluvoxamine on fatigue in these patients with cholestatic liver disease and severe chronic fatigue.

\section{Background}

Primary biliary cirrhosis (PBC) and primary sclerosing cholangitis (PSC) are chronic cholestatic liver diseases with a relatively favourable prognosis for most patients $[1,2]$. Fatigue is now recognised as a major clinical problem in PBC, with a reported prevalence of up to $85 \%$ [3]. Although fatigue has not been studied extensively in PSC, it is a problem in many of these patients as well [4]. There have been no reports of drugs or other treatment modalities with a beneficial effect on fatigue associated with these diseases, and although the widely used drug ursodeoxycholic acid improves the biochemical abnormalities in these diseases, an effect on fatigue has not been shown $[5,6]$. 
Recent studies suggest that $45-72 \%$ of patients may have a depressive disorder [3,5]. Fatigue is a frequent symptom of depression. We hypothesised that common mechanisms may be involved in the aetiology of both fatigue and depression or that fatigue could be a symptom of a depressive disorder in PBC and PSC. If this would be true, antidepressive drugs could have a beneficial effect on fatigue and potentially also on overall quality of life. Thus, the present study aimed to evaluate the effects of an antidepressant on fatigue and quality of life in PBC and PSC. The antidepressant fluvoxamine (a selective serotonin reuptake inhibitor, SSRI) was chosen because of several reasons. First, fluvoxamine is one of the oldest SSRI's, and the risk of unexpected side-effects was therefore low. Second, there was extensive local experience with this particular drug. Third, there is a documented correlation between serum concentrations and effects for fluvoxamine, which could be used in future studies using this drug.

\section{Methods \\ Participants}

All patients visiting the outpatient clinic of our hospital with a diagnosis of PBC or PSC and self-reported significant fatigue for which the patient was willing to receive treatment were invited to participate. Patients with a diagnosis of depression or other psychiatric disorder, any change in medication in the past three months, incomplete understanding of the Dutch language, pregnancy, current lactation or lack of adequate contraception, ChildPugh score of $\geq 6$ or receiving other study medication in the preceding 6 months were excluded. The Beck Depression Inventory was used to screen for the presence of depression, and in patients with a score $>10$ a psychiatric evaluation using the SADS DSM-IV was performed [7]. Written informed consent was obtained in all patients. The institutional review board approved the study.

\section{Treatment}

Patients were treated with identical capsules containing $75 \mathrm{mg}$ of fluvoxamine or placebo. They were instructed to take a capsule in the evening before sleeping on the first two days, and to add one capsule in the morning starting on day three and to continue this treatment regimen of 75 mg BID for a total period of six weeks. This dose is a widely used dose for the treatment of depression. Since previous data on the effects on fatigue were lacking, this dose was used in the present study. Patients were instructed to contact the trial co-ordinators in case of side effects.

\section{Objectives}

The aim of the study was to assess the efficacy of fluvoxamine in the treatment of fatigue associated with $\mathrm{PBC}$ or PSC.

\section{Outcomes}

The primary outcome was an improvement in fatigue, as measured by a visual analogue scale (VAS), the Fisk Fatigue Severity Scale (FFSS) [8] and the Multidimensional Fatigue Inventory (MFI) [9]. The FFSS has been previously validated for assessing fatigue in PBC [10]. The VAS consisted of a $10-\mathrm{cm}$ line with on one end 'no fatigue at all during the last month' and on the other end 'extreme fatigue during the last month'. Patients were asked to indicate the severity of fatigue by placing a marking on the line. A higher score represents increased fatigue in these three outcome measures. Secondary outcomes were changes in quality of life, assessed by the SF-36 questionnaire [11], changes in pruritus using a VAS and changes in laboratory tests. The VAS for pruritus consisted of a $10-\mathrm{cm}$ line with on one end 'no pruritus at all during the last month' and on the other end 'severe pruritus during the last month'. At entry and after 42 days the following tests were performed: FFSS, MFI, SF-36, VAS for fatigue and pruritus, total serum bilirubin, albumin, gamma-GT, alkaline phosphatase (APh), alanine aminotransferase (ALT), aspartate aminotransferase (AST), cholesterol, immunoglobulin G (IgG), immunoglobulin M (IgM), prothrombin time (PT), antithrombin-III (AT-III), haemoglobin, leukocyte and platelet count. At 14 days, the FFSS, MFI and serum bilirubin, ALT and APh were repeated. At day 70, the MFI, FFSS and the VAS for fatigue and pruritus were obtained.

\section{Sample Size}

A formal power calculation was precluded by absence of data on the efficacy of fluvoxamine in the treatment of fatigue associated with PBC or PSC. In order to detect an effect similar to the expected therapeutic effect of fluvoxamine in depression (25\% improvement in the placebogroup and $75 \%$ in the fluvoxamine-group), with a power of $80 \%$ and assuming a drop-out rate of $5 \%$, it was calculated that 40 patients needed to be recruited (Stata 5.0, Stata Corporation, College Station, Texas, USA).

\section{Randomisation}

Patients were allocated to a serial number (by authors PB or $\mathrm{HB}$ ) corresponding to a previously prepared package of capsules. The capsules containing fluvoxamine or placebo were prepared by the hospital pharmacy. The packages were numbered according to a computer generated randomisation list prepared by the trial statistician $(\mathrm{BH})$. Thus, both patients and those assessing the outcomes were blinded to group assignment. Patients were stratified for disease (PBC or PSC). Opaque, closed envelopes containing information on the allocated treatment for each patient number were prepared for medical emergencies. 


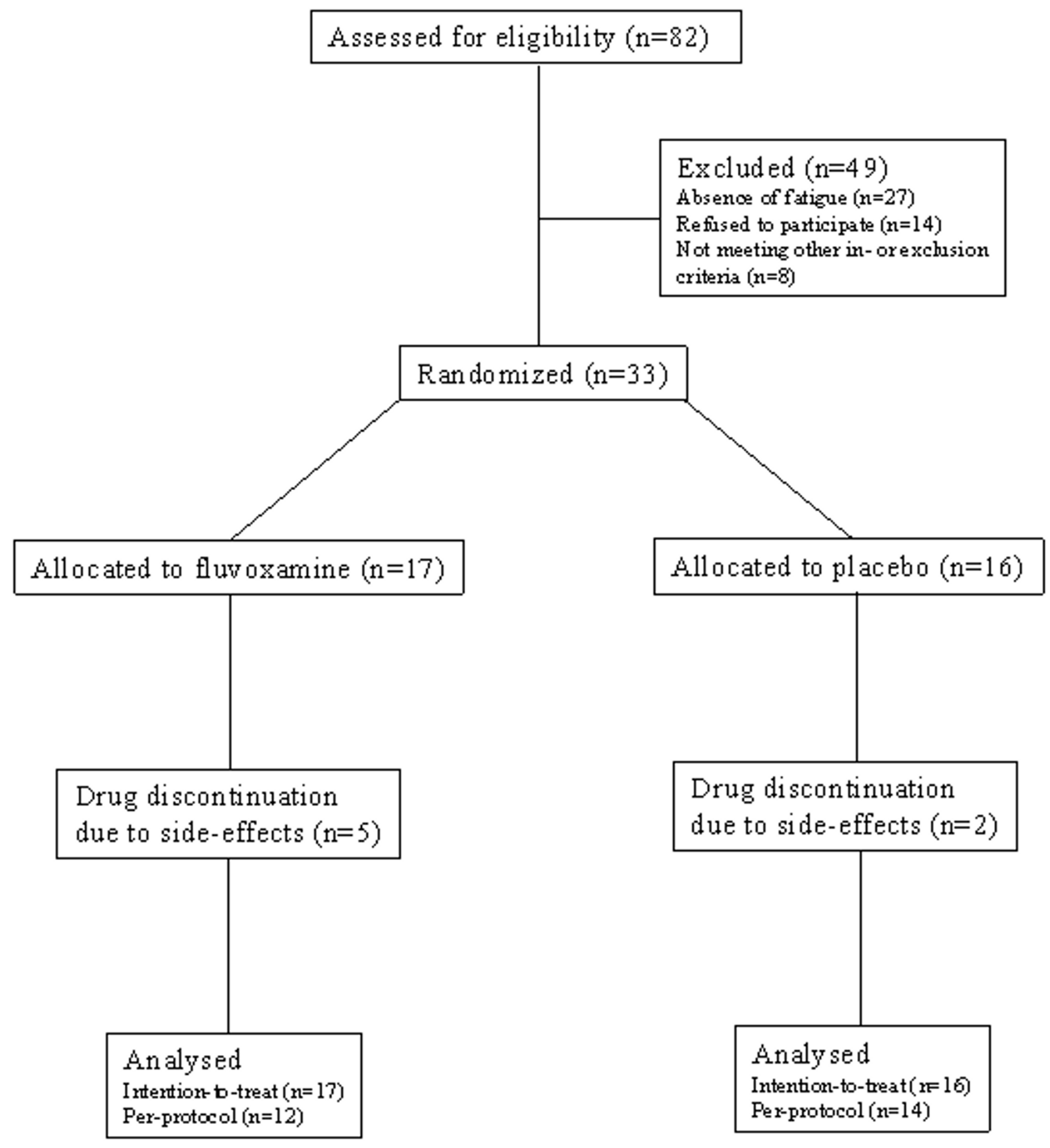

Figure I

Trial profile 


\section{Statistical methods}

Data were analysed according to both the intention-totreat and the per-protocol principle. Differences between groups were planned to be tested with the $\chi^{2}$ and Student's t-test. However, in part because of the lower than expected patient recruitment and the higher than expected dropout rate, a normal data distribution was not present for most variables. Therefore, it was decided that the MannWhitney test would be used for testing differences between groups. Statistical tests were two-tailed. P-values of 0.05 or less were considered statistically significant. In the analyses where the multiple time points were compared, a p-value of $<0.05$ divided by 3 ( $p<0.017$ ) was considered statistically significant. Subgroup analyses including only patients with PBC and PSC were performed using the same statistical methods. Statistical analyses were performed using SPSS (Version 9.0, SPSS Inc, Chicago, IL, U.S.A).

\section{Results}

\section{Recruitment, allocation and participants flow}

Patients were included between October 2001 and June 2002. Five patients (29\%) in the fluvoxamine group and 2 patients (13\%) in the placebo group discontinued treatment because of side effects $(\mathrm{p}=0.40)$. All randomised patients were included in the intention-to-treat analysis, and all patients completing the six weeks treatment period were included in the per-protocol analysis (Figure 1).

\section{Baseline characteristics}

Of the 82 patients screened for inclusion, 33 met all inclusion and exclusion criteria and gave their informed consent to participate in the trial. Although 18/33 (55\%) patients had a Beck Depression Inventory score $>10$ points, suggesting the presence of depression, additional psychiatric evaluation (EO and $\mathrm{WB}$ ) according to the DSM-IV found no evidence for depression in any of these cases. In three patients not suitable for inclusion in the present trial a diagnosis of depression was made according to the DSM-IV criteria. One patient had been diagnosed with depression thirteen years before entry; however psychiatric evaluation found no evidence for depression at the time of entry. Of the 33 patients, 16 were allocated to the placebo group and 17 to the fluvoxamine group. General baseline data and questionnaire results are shown in tables 1 and 2, respectively. All patients had been fatigued during at least two years; none had renal failure, chronic anaemia or serum electrolyte abnormalities. One patient (fluvoxamine group) was receiving treatment for hypothyroidism and was already fatigued before thyroid dysfunction developed and thyroid function was documented to be normal. In all other patients thyroid function was normal. Five patients ( 4 in the fluvoxamine group) were using $\beta$-blockers; in all cases chronic fatigue had been present before initiation of this therapy. Patients in the fluvoxamine group had significantly higher scores in the cognitive and social domains of the FFSS ( $p=0.04$ for both domains). Differences for all other baseline variables were non-significant.

\section{Outcomes}

Analysis on intention-to-treat basis (17 and 16 patients in the fluvoxamine and placebo group, respectively) yielded no significant differences in response between the fluvoxamine and the placebo group with respect to the primary outcome measures, the fatigue visual analogue score, MFI and FFSS (Table 3 ). Per-protocol analysis (12 patients and 14 patients in the fluvoxamine and placebo group, respectively) of the same outcomes showed no significant differences except for a borderline significant difference in the general fatigue domain of the MFI in favour of the placebo group $(\mathrm{p}=0.04)$. The results of the intention-to-treat analysis with respect to the fatigue VAS scores are shown in figure 2. Analysis (intention-to-treat) of changes in SF-

Table I: Baseline characteristics (general)

\begin{tabular}{llll}
\hline & Fluvoxamine $N=17$ & Placebo $N=16$ & P-value \\
\hline Male/Female & $4 / 13$ & $6 / 10$ & 0.38 \\
PBC/PSC & $12 / 5$ & $10 / 6$ & 0.57 \\
Age (median) & 51 & 54 & 0.38 \\
Pruritus (yes/no) & $9 / 8$ & $8 / 8$ & 0.87 \\
UDCA dose (median, mg/day) & 750 & 900 & 0.14 \\
Previous depressive disorder (yes/no) & $(0 / 17)$ & $(1 / 15)$ & 0.30 \\
Weight (median, kg) & 67 & 71 & 0.95 \\
Length (median, cm) & 173 & 171 & 0.67 \\
Body Mass Index (median) & 24.2 & 24.6 & 0.61 \\
Haemoglobin (mmol/l, median) & 8.3 & 8.8 & 0.21 \\
Bilirubin ( $\mu$ mol/l, median) & 13 & 11.5 & 0.83 \\
Alkaline Phosphatase (U/l, median) & 137 & 192 & 0.36 \\
ALT (U/l, median) & 42 & 43 & 0.55 \\
Albumin (U/l, median) & 42 & 41.5 & 0.81
\end{tabular}

Laboratory reference values: haemoglobin 7.5-9.5 mmol/l, bilirubin 0-16 $\mu \mathrm{mol} / \mathrm{l}$, alkaline phosphatase 0-I I9 U/l, ALT 0-30 U/l, albumin 35-50 g/l. 
Table 2: Baseline values of outcome measures

\begin{tabular}{llll}
\hline & Fluvoxamine $(\mathrm{n}=17)$ & Placebo $(\mathrm{n}=16)$ & P-value \\
\hline VAS Fatigue & 7.40 & 7.45 & 0.91 \\
VAS Pruritus & 3.50 & 1.15 & 0.33 \\
Beck Depression Inventory & 11.0 & 10.5 & 0.45 \\
FFSS & & & 0.25 \\
$\quad$ Physical Domain & 24.0 & 16.5 & 0.04 \\
$\quad$ Cognitive Domain & 15.0 & 9.0 & 0.04 \\
Social Domain & 37.0 & 22.5 & 0.78 \\
MFI & & & 0.22 \\
General Fatigue & 18.0 & 17.5 & 0.30 \\
Physical Fatigue & 16.0 & 14.0 & 0.17 \\
$\quad$ Reduction in Activity & 15.0 & 13.0 & 0.90 \\
Reduction in Motivation & 13.0 & 10.0 & 0.47 \\
Mental Fatigue & 13.0 & 13.0 & 0.35 \\
SF-36 & & & 0.55 \\
Physical Functioning & 65.0 & 70.0 & 0.63 \\
Role Functioning Physical & 25.0 & 25.0 & 0.64 \\
Bodily Pain & 62.0 & 62.0 & 0.34 \\
General Health & 40.0 & 35.0 & 0.46 \\
Vitality & 45.0 & 40.0 & 0.28 \\
Social Functioning & 62.5 & 62.5 & 1.00 \\
Role Emotional Functioning & 100.0 & 66.7 & 60.0 \\
Mental Health & 56.0 & 4.0 & \\
Reported Health Transition & 4.0 & & \\
\hline
\end{tabular}

All results are expressed as median scores.

Table 3: Primary outcomes, change from baseline, intention-to-treat

\begin{tabular}{|c|c|c|c|c|c|c|c|c|c|}
\hline & \multicolumn{3}{|c|}{ Placebo $(n=16)$} & \multicolumn{3}{|c|}{ Fluvoxamine $(n=17)$} & \multicolumn{3}{|l|}{ P-value } \\
\hline & Day 14 & Day 42 & Day 70 & Day 14 & Day 42 & Day 70 & Day 14 & Day 42 & Day 70 \\
\hline VAS Fatigue & -1.3 & -0.6 & -0.55 & -0.3 & -0.4 & -0.35 & 0.31 & 0.51 & 0.51 \\
\hline \multicolumn{10}{|l|}{ FFSS } \\
\hline Physical Domain & -2.0 & -3.5 & -4.0 & 0 & -2.5 & 0 & 0.31 & 0.40 & 0.12 \\
\hline Cognitive Domain & -1.5 & 1.0 & 0 & -1.0 & -1.0 & 0 & 0.69 & 0.85 & 0.60 \\
\hline Social Domain & -3.0 & -3.5 & -2.0 & -3.0 & -4.0 & -1.5 & 0.66 & 0.93 & 0.93 \\
\hline \multicolumn{10}{|l|}{ MFI } \\
\hline General Fatigue & -2.0 & 0 & 0 & 0 & 0 & -1.0 & 0.10 & 0.62 & 0.87 \\
\hline Physical Fatigue & 0 & 0 & 0 & 0 & 1.0 & 1.0 & 0.92 & 0.73 & 0.44 \\
\hline Reduction Activity & 0.5 & -1.0 & 0 & 1.0 & -0.5 & 1.0 & 0.20 & 0.56 & 0.13 \\
\hline Reduction Motivation & -1.0 & 0 & -1.0 & 1.0 & -0.5 & 1.0 & 0.08 & 0.94 & 0.71 \\
\hline Mental Fatigue & -1.0 & -0.5 & 0 & 0 & 0 & 0 & 0.06 & 0.35 & 0.89 \\
\hline
\end{tabular}

Results are expressed as median differences in scores compared to baseline.

36 scores revealed no differences between treatment groups. No significant difference in pruritus was found according to VAS scores at entry and at 6 weeks in either group. The only (borderline) significant difference in the laboratory test results was for serum alkaline phosphatase at day 42 and day 0 , attributable to a significant decrease at 42 days in the placebo group $(\mathrm{p}=0.03)$. Subgroup anal- ysis of only patients with PBC or PSC showed no significant differences between the fluvoxamine and placebo groups (Tables 4 and 5). In addition, no significant differences in reponse were found between men and women.

One patient treated with fluvoxamine who suffered from fatigue for many years prior to inclusion in the present 


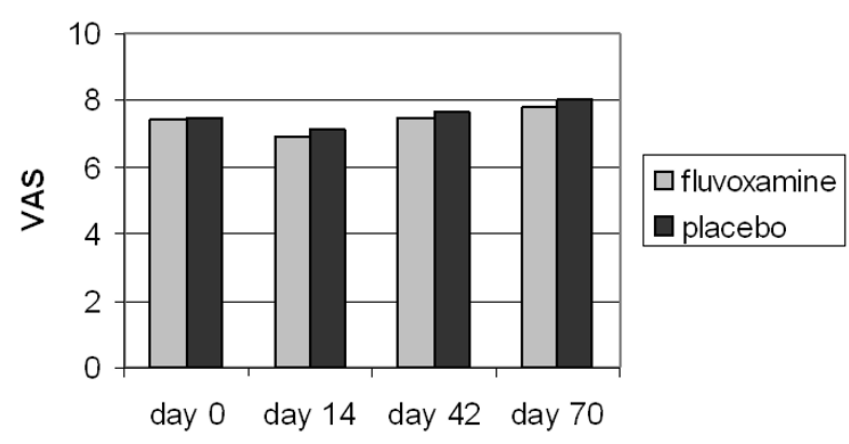

Figure 2

Visual analogue scale for fatigue. Median Visual Analogue Scores for fatigue. No significant differences were found within and between groups at entry, during treatment (day 14 and day 42) and 4 weeks after stopping the trial medication.

trial reported a substantial improvement in fatigue during treatment, and a subsequent deterioration after treatment discontinuation. It was decided to continue fluvoxamine treatment in this patient and she has currently been free of fatigue for two years. Her VAS scores for fatigue were 8.4 prior to treatment, 6.9 after 14 days and 2.5 after 6 weeks. Four weeks after treatment discontinuation, her VAS score for fatigue was 7.6.

\section{Adverse events}

Treatment was discontinued due to side effects in 5 patients in the fluvoxamine group (after 2, 5, 5, 6 and 7 days) and 2 patients (after 7 and 28 days in the placebo group. These side effects were headache, nausea (2 patients), insomnia and dizziness (3 patients) in the fluvoxamine group, and diarrhoea $(n=2)$ in the placebo group. All side effects resolved rapidly after discontinuation of the treatment and none required specific therapy or hospitalisation.

\section{Discussion}

In the present study no beneficial effect of the antidepressant drug fluvoxamine in the treatment of fatigue associated with PBC and PSC could be demonstrated. Further, given the results of quality of life assessment, no evidence was found for an effect on overall well being. There are several potential explanations for the negative outcome of this trial. The most logical would be that fatigue is neither a symptom of a depressive disorder commonly present in PBC and PSC nor that the aetiology of fatigue and depression are interrelated, sharing a common pathway that could be modified by anti-depressive medications. We believe this is the most likely explanation. Although a high prevalence of depression has been reported in PBC
$[3,12]$, we doubt whether this also applies to our patients as preliminary results of an ongoing study indicate that depression in our patients with PBC and PSC is infrequent (study in progress). On the other hand, the trial may have failed to detect a true therapeutic effect of fluvoxamine. The trial was designed to detect a large treatment effect while the number of patients that was enrolled in the study was lower than expected. In addition, the number of patients discontinuing treatment was higher than expected. Therefore, a (small) beneficial effect of fluvoxamine may well have been missed, and larger trials using fluvoxamine or another SSRI may be needed to define the efficacy of these drugs in the treatment of fatigue. However, the initiation of large trials in cholestatic liver disease is particularly difficult since these diseases are relatively rare. As a result international collaboration is usually needed but acquiring sufficient funding for such collaboration is a recurrent problem.

Although on the whole patient groups were nicely balanced, the baseline scores in the cognitive and social domains of the FFSS were higher for the fluvoxamine group, suggesting fatigue might have been more severe in this group. However, since all other measurements of fatigue showed no significant differences, it appears unlikely that bias due to differences in base-line characteristics of the treatment groups has significantly influenced the results. Finally, the duration of treatment may have been too short, given the chronic character of fatigue associated with cholestatic liver diseases. Although therapeutic effects of fluvoxamine when used for treating depression are in general apparent within 2-4 weeks, the possibility that therapeutic effects may only become apparent after more prolonged treatment cannot be excluded.

Side effects leading to discontinuation of the drug occurred in one third of patients receiving fluvoxamine treatment. This was much more frequent than expected and contrasting with available data on the tolerability of the drug. We have no clear suggestion as to a possible explanation. The $150 \mathrm{mg}$ dose we used was comparable to those used in other studies and within the $100-200 \mathrm{mg}$ dose range frequently used for treating depression. This experience raises the possibility that patients with cholestatic disorders are more susceptible for developing side effects. Unfortunately, we did not monitor plasma fluvoxamine concentrations and therefore cannot exclude the possibility that side effects were related to higher fluvoxamine blood concentrations as compared to those in normal individuals. In this context it should be noted that all patients were ambulant and had relatively mild liver dysfunction. None of the side effects required intervention or persisted after discontinuation of the drug, and there were no obvious deteriorations in liver tests in any of the patients. There have been no previous studies of 
Table 4: Primary outcomes for patients with PBC

\begin{tabular}{|c|c|c|c|c|c|c|c|c|}
\hline & \multicolumn{4}{|c|}{ Placebo $(n=10)$} & \multicolumn{4}{|c|}{ Fluvoxamine $(n=12)$} \\
\hline & Day 0 & Day 14 & Day 42 & Day 70 & Day 0 & Day 14 & Day 42 & Day 70 \\
\hline VAS Fatigue & 7.6 & 6.9 & 7.9 & 7.5 & 6.8 & 6.9 & 8.8 & 7.6 \\
\hline \multicolumn{9}{|l|}{ FFSS } \\
\hline Physical Domain & 20 & 18 & 18 & 15 & 16 & 16 & 21 & 21 \\
\hline Cognitive Domain & 9 & 6 & 14 & 8 & 11 & 8 & 10 & 9 \\
\hline Social Domain & 25 & 26 & 28 & 19 & 26 & 20 & 22 & 15 \\
\hline \multicolumn{9}{|l|}{ MFI } \\
\hline General Fatigue & 17 & 15 & 17 & 16 & 16 & 16 & 17 & 16 \\
\hline Physical Fatigue & 14 & 14 & 15 & 16 & 14 & 13 & 14 & 14 \\
\hline Reduction Activity & 13 & 13 & 13 & 11 & 12 & 14 & 14 & 15 \\
\hline Reduction Motivation & 9 & 9 & 12 & 11 & 11 & 13 & 11 & 12 \\
\hline Mental Fatigue & 14 & 12 & 12 & 14 & 13 & 14 & 14 & 12 \\
\hline
\end{tabular}

Results are expressed as median scores.

Table 5: Primary outcomes for patients with PSC

\begin{tabular}{|c|c|c|c|c|c|c|c|c|}
\hline & \multicolumn{4}{|c|}{ Placebo $(n=6)$} & \multicolumn{4}{|c|}{ Fluvoxamine $(n=5)$} \\
\hline & Day 0 & Day 14 & Day 42 & Day 70 & Day 0 & Day 14 & Day 42 & Day 70 \\
\hline VAS Fatigue & 7.3 & 7.4 & 7.5 & 8.3 & 6.4 & 6.8 & 7.5 & 7.9 \\
\hline \multicolumn{9}{|l|}{ FFSS } \\
\hline Physical Domain & 14 & 13 & 9 & 9 & 28 & 27 & 26 & 28 \\
\hline Cognitive Domain & 8 & 11 & 8 & 7 & 20 & 20 & 19 & 18 \\
\hline Social Domain & 17 & 12 & II & 13 & 48 & 49 & 47 & 48 \\
\hline \multicolumn{9}{|l|}{ MFI } \\
\hline General Fatigue & 18 & 18 & 17 & 17 & 19 & 19 & 19 & 20 \\
\hline Physical Fatigue & 13 & 15 & 14 & 13 & 18 & 19 & 19 & 19 \\
\hline Reduction Activity & 12 & 12 & II & 12 & 18 & 18 & 17 & 20 \\
\hline Reduction Motivation & 10 & 10 & 8 & 8 & 18 & 17 & 18 & 16 \\
\hline Mental Fatigue & 12 & 10 & 10 & 11 & 14 & 14 & 13 & 13 \\
\hline
\end{tabular}

Results are expressed as median scores.

fluvoxamine or other antidepressants in the treatment of fatigue associated with cholestatic liver diseases, but several studies have addressed the efficacy of these drugs in the treatment of chronic fatigue syndrome and fibromyalgia. According to a recent meta-analysis, antidepressants are effective in the treatment of many of the symptoms of fibromyalgia, including fatigue [13]. Two randomised controlled trials, however, did not find an improvement in patients with the chronic fatigue syndrome $[14,15]$.

\section{Conclusions}

In conclusion, although the present study lacked power to detect small but possibly relevant treatment effects, no evidence for a therapeutic effect of fluvoxamine in the treatment of fatigue associated with PBC or PSC was found. The results may indirectly suggest that fatigue is not a symptom of an underlying depressive disorder in these patients with cholestatic liver disease.

\section{Competing interests}

None declared.

\section{Authors' contributions}

All authors participated in study design and read and approved the final manuscript. PB and HB initiated and coordinated the study, performed patient recruitment and inclusion and prepared the manuscript. EO and WB performed the psychiatric evaluations. $\mathrm{PB}, \mathrm{HB}$ and $\mathrm{BH}$ participated in the statistical analysis.

\section{Acknowledgements}

A grant was provided by the Gastrostart foundation.

\section{References}

I. van Hoogstraten HJF, Hansen BE, van Buuren HR, ten Kate FJW, van Berge Henegouwen GP, Schalm SW: Prognostic factors and longterm effects of ursodeoxycholic acid on liver biochemical parameters in patients with primary biliary cirrhosis. J Hepatol 1999, 31:256-262. 
2. Broome U, Olsson R, Loof L, Bodemar G, Hultcrantz R, Danielsson A, Prytz H, Sandberg-Gertzen H, Wallerstedt S, Lindberg G: Natural history and prognostic factors in 305 Swedish patients with primary sclerosing cholangitis. Gut 1996, 38:610-615.

3. Huet PM, Deslauriers J, Tran A, Faucher C, Charbonneau J: Impact of fatigue on the quality of life of patients with primary biliary cirrhosis. Am J Gastroenterol 2000, 95:760-767.

4. Wiesner RH, LaRusso NF, Ludwig J, Dickson ER: Comparison of the clinicopathologic features of primary sclerosing cholangitis and primary biliary cirrhosis. Gastroenterology 1985, 88: $108-1 \mid 4$.

5. Gluud C, Christensen E: Ursodeoxycholic acid for primary biliary cirrhosis. Cochrane Database Syst Rev 2002:CD00055I..

6. van Hoogstraten HJF, Wolfhagen FHJ, van de Meeberg PC, Kuiper H, Nix GAJ], Becx MCJM, Hoek AC, van Houte DPF, Rijk MCM, Salemans JMJI, Scherpenisse J, Schrijver M, Smit AM, Spoelstra P, Stadhouders PHGM, Tan TG, Hop WCJ, ten Kate FJW, van Berge Henegouwen GP, Schalm SW, van Buuren HR: Ursodeoxycholic acid therapy for primary sclerosing cholangitis: results of a 2year randomized controlled trial to evaluate single versus multiple daily doses. J Hepatol I998, 29:4I7-423.

7. Beck AT, Ward CH, Mendelson M: An inventory for measuring depression. Arch Gen Psychiatry 196I, 6:56I-57I.

8. Fisk JD, Ritvo PG, Ross L, Haase DA, Marrie TJ, Schlech WF: Measuring the functional impact of fatigue: initial validation of the fatigue impact scale. Clin Infect Dis 1994, I 8 Suppl I:S79-83.

9. Smets EM, Visser MR, Willems-Groot AF, Garssen B, Oldenburger F, van Tienhoven G, de Haes JC: Fatigue and radiotherapy: (A) experience in patients undergoing treatment. $\mathrm{Br} J$ Cancer I998, 78:899-906.

10. Prince MI, James OF, Holland NP, Jones DE: Validation of a fatigue impact score in primary biliary cirrhosis: towards a standard for clinical and trial use. J Hepatol 2000, 32:368-373.

II. Ware J. E., Jr., Sherbourne CD: The MOS 36-item short-form health survey (SF-36). I. Conceptual framework and item selection. Med Care 1992, 30:473-483.

12. Cauch-Dudek K, Abbey S, Stewart DE, Heathcote E): Fatigue in primary biliary cirrhosis. Gut 1998, 43:705-7I0.

13. O'Malley PG, Balden E, Tomkins G, Santoro J, Kroenke K, Jackson JL: Treatment of fibromyalgia with antidepressants: a metaanalysis. J Gen Intern Med 2000, 15:659-666.

14. Vercoulen JH, Swanink CM, Zitman FG, Vreden SG, Hoofs MP, Fennis JF, Galama JM, van der Meer JW, Bleijenberg G: Randomised, double-blind, placebo-controlled study of fluoxetine in chronic fatigue syndrome. Lancet 1996, 347:858-86I.

15. Wearden AJ, Morriss RK, Mullis R, Strickland PL, Pearson DJ, Appleby L, Campbell IT, Morris JA: Randomised, double-blind, placebocontrolled treatment trial of fluoxetine and graded exercise for chronic fatigue syndrome. BrJ Psychiatry 1998, 172:485-490.

\section{Pre-publication history}

The pre-publication history for this paper can be accessed here:

http://www.biomedcentral.com/1471-230X/4/13/pre pub

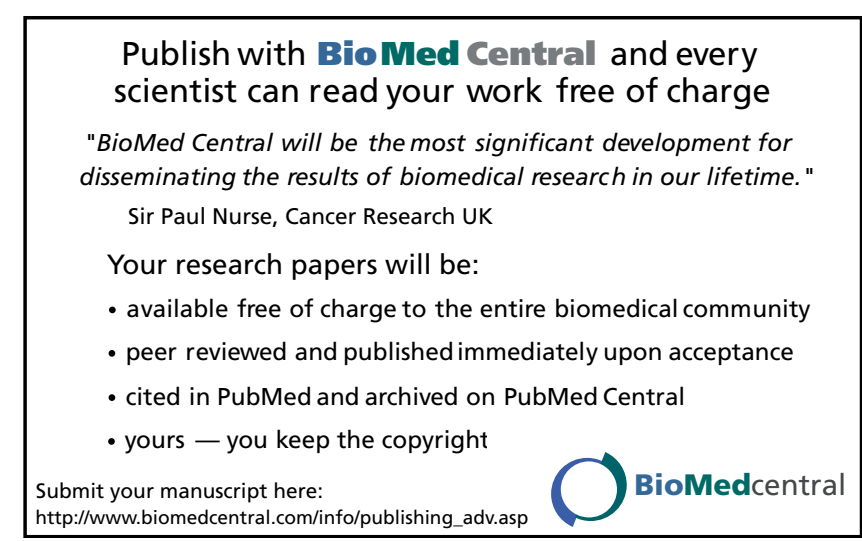

